\title{
Detection of the pan neuronal marker PGP9.5 by immuno- histochemistry and quantitative PCR in eutopic endometrium from women with and without endometriosis
}

\author{
Héctor Barrera-Villa Zevallos • Brett McKinnon • \\ Natsuko Tokushige $\cdot$ Michael D. Mueller • \\ Ian S. Fraser · Nick A. Bersinger
}

Received: 28 February 2014 / Accepted: 9 July 2014/Published online: 22 July 2014

(c) Springer-Verlag Berlin Heidelberg 2014

\begin{abstract}
Purpose To assess endometrial gene as well as protein expression of neuroendocrine and supposedly endometriosis-associated product PGP9.5 and pain symptoms in women with endometriosis and controls undergoing laparoscopy, using molecular biological and immuno-histochemical approaches in the same patients.

Methods Biopsy of eutopic endometrium from 29 patients by sharp curettage, and preparation of paraffin blocks. Determination of PGP9.5 gene expression and protein abundance using qPCR and immuno-histochemistry.

Results qPCR; The PGP9.5 mRNA expression level between women with $(N=16)$ and without $(N=13)$ endometriosis was not different, regardless of pain symptoms or menstrual cycle phase. PGP9.5 expression was higher in women who reported pain compared to those who did not; however, this association was not statistically
\end{abstract}

H. B.-V. Zevallos · I. S. Fraser

Department of Obstetrics, Gynaecology and Neonatology, Queen Elizabeth II Research Institute for Mothers and Infants, University of Sydney, Sydney, Australia

H. B.-V. Zevallos

Universidad Anáhuac, México City, Mexico

B. McKinnon - N. A. Bersinger

Department of Clinical Research, University of Berne, Bern, Switzerland

N. Tokushige

Pain Management Research Institute, Kolling Institute of Medical Research, University of Sydney, Sydney, Australia

M. D. Mueller · N. A. Bersinger ( $₫)$

Department of Obstetrics and Gynaecology, University of Berne, DKF Murtenstrasse 35, 3010 Berne, Switzerland

e-mail: nick.bersinger@dkf.unibe.ch significant. The expression of PGP9.5 mRNA was higher in women with endometriosis and pain during the proliferative than in the secretory phase $(P=0.03)$. Furthermore, in the first half of the cycle, the abundance of the PGP9.5 transcript was also significantly higher in endometriosis patients compared to those without $(P=0.03)$. Immunohistochemistry; Thirteen of the 16 endometriosis patients showed positive PGP9.5 immuno-reactivity in the endometrium, whereas no such signal was observed in women without endometriosis. The absolute number of nerve fibres per $\mathrm{mm}^{2}$ in women with endometriosis was similar, regardless of the pain symptoms.

Conclusions PGP9.5 mRNA expression is increased in the proliferative phase of endometriotic women with pain. The presence of nerve fibres was demonstrated by a PGP9.5 protein signal in immuno-histochemistry and restricted to patients with endometriosis. Based on these results, however, there did not appear to be a direct association between the gene expression and protein abundance in women with and without endometriosis or those that experienced pain.

Keywords Endometriosis - PGP9.5 expression - Eutopic endometrium $\cdot$ Pain $\cdot$ Nerve fibres

\section{Introduction}

Endometriosis is characterised by the presence of lesions composed of endometrial-like glands and stroma in the peritoneal cavity. It is considered a non-malignant condition; however, it does share some components with malignancy, such as angiogenesis, abnormal apoptotic patterns, unrestrained cellular proliferation and rare invasion of distant organs (e.g., lungs, pleura and brain) [1-3]. 
Chronic pelvic pain is the most distressing symptom in most women with endometriosis. It is not only related to menstrual discomfort, it also affects other aspects of patient's everyday quality of life, such as sexual intercourse, emotional interaction, social activity, family relations and work performance. The mechanisms generating pain in women with endometriosis are not completely understood. The spectrum of symptoms characteristic of endometriosis could be explained by nociceptive transmission induced by inflammatory mediators produced by numerous cell types in women with endometriosis, which might be associated with the pain symptoms linked with visceral sensitisation [4]. Furthermore, profuse infiltration of nociceptors on sensory $\mathrm{A} \delta$ and $\mathrm{C}$ fibres (peptidergic fibres NTRK1 and SP positive) has been reported in deep infiltrating endometriosis from the cul-de-sac, uterosacral ligament and intestinal lesions [5].

In recent years, the findings of Tokushige et al. [6-8] and Al-Jefout et al. [9, 10] regarding the presence of protein gene product 9.5 (PGP9.5) positive nerve fibres in the functional layer of the endometrium of some women with endometriosis, but never in those without the disease, and in endometriotic lesions have promoted a growing interest in this area. PGP9.5 (also known as ubiquitin carboxylterminal esterase L1, UCHL1) is a cytoplasmic thiolesterase restricted to nervous and neuroendocrine cells and it is involved in nerve cell differentiation, neuronal ubiquitination, ubiquitin ligation to target proteins for proteolysis, ubiquitin detachment from ubiquitin chains, apoptosis and cell cycle regulation [11]. PGP9.5 is a C-terminal hydrolase that dissociates ubiquitin peptidic bonds and thus regulates proteolysis mediated by proteasomes and ubiquitin signalling $[12,13]$. This de-ubiquitinase, like many others, helps to regulate the balance of protein degradation by detaching ubiquitin from specific substrates and by recycling it, increasing in this way its availability for the destruction of other proteins [14].

Given that it has been suggested that PGP9.5 might be involved in degradation processes and possible regulation of neuronal lifespan and differentiation [15] we decided to investigate a possible correlation between the endometrial expression of the PGP9.5 gene and pain symptoms in women with endometriosis, using quantitative polymerase chain reaction (qPCR) to assess the levels of transcription, and immuno-histochemistry (IHC) to confirm post-transcriptional expression.

\section{Materials and methods}

Tissue collection and patient details

Endometrial tissue samples were collected by sharp curettage (using a 6 or $7 \mathrm{~mm}$ curette); two to three strips from each subject were formalin fixed and paraffin embedded (FFPE) for the study and the remainder was sent for routine pathology assessment. Twenty-nine FFPE endometrial tissue samples were obtained from women with $(N=16)$ and without $(N=13)$ peritoneal endometriosis. Biopsies from patients confirmed to have no endometriosis were collected from women who had undergone laparoscopy due to unexplained infertility. The same specimens were used for RNA and protein analyses in an effort to directly compare gene expression and protein abundance in the same sample. The tissue samples were divided into four subgroups: (1) endometriosis patients with pelvic pain $(N=9$, four in proliferative and five in secretory phases), (2) endometriosis patients without pelvic pain $(N=7$, two in proliferative and five in secretory phases), (3) disease free women with pain $(N=7$, four in proliferative and three in secretory phases), and (4) diseasefree women without pelvic pain $(N=6$, three in both proliferative and secretory phases each). None of the participants was under hormonal treatment. Ethics approval was obtained from the Institutional Ethics Committees from the Royal Prince Alfred Hospital, Sydney and the University of Sydney. Patients were recruited prior to admission for laparoscopic surgery and gave fully informed and signed consent to participation. The researchers were blinded to the diagnosis until laboratory data were analysed.

\section{RNA isolation and qPCR}

The tissues were cut at $12 \mu \mathrm{m}$ for total RNA extraction. RNA was isolated after scraping, dewaxing with xylene and rehydrating with $100 \%$ ethanol using the RNeasy FFPE Kit (Qiagen). RNA of sufficient concentration and spectroscopic quality was obtained from all samples except two, and $250 \mathrm{ng}$ of each were reverse transcribed. A pre-amplification of the cDNA was performed prior to gene expression analysis with the TaqMan PreAmp Master Mix and a pooled mixture of all subsequent gene expression assays at $0.2 \times$ concentration. Subsequently, real-time PCR was performed, after dilution, with the TaqMan Fast Advanced Master Mix using a Corbett Rotor-Gene RG-2000 cycler for GAPDH, HPRT1 and ACTB as housekeeping genes, and for PGP9.5 as the gene of interest. All amplicon sizes ranged between 60 and $84 \mathrm{bp}$. Table 1 shows the sequences of the primers used. No template controls were included for RT and for each qPCR run. The raw data were analysed with the Biogazelle software (Biogazelle, Zwijnaarde, Belgium) and gene expression for each individual sample was defined as the fold change from an arbitrary calibrator sample. A $P$ value of less than 0.05 was considered significant. 
Immuno-histochemistry

The FFPE tissue blocks were cut at $4 \mu \mathrm{m}$ thickness, mounted onto SuperFrost Ultra Plus microscope slides (Menzel Glaser-Braunschweig, Germany) and dried at $60{ }^{\circ} \mathrm{C}$ for $1 \mathrm{~h}$. Dried slides were deparaffinised through xylene and descending concentrations of alcohol to water. Deparaffinised slides for immuno-histochemical staining underwent antigen retrieval for $20 \mathrm{~min}$ in pre-heated $100{ }^{\circ} \mathrm{C} \mathrm{pH} 9.0$ target retrieval solution (Dako, Denmark A/S, Denmark). Slides were cooled for $30 \mathrm{~min}$ and rinsed in a tris-buffered saline (TBS) bath in two changes and incubations of $5 \mathrm{~min}$ each. The immuno-histochemical staining was performed using the Dako Autostainer Model S3400 (Dako, Carpinteria, CA, USA). A $3 \%$ hydrogen peroxide block (Dako A/S, Denmark) was applied for

Table 1 Primers used for qRT-PCR

\begin{tabular}{llll}
\hline Gene & $\begin{array}{l}\text { Gene Bank } \\
\text { accession number }\end{array}$ & $\begin{array}{l}\text { TaqMan gene } \\
\text { expression assay } \\
\text { number }\end{array}$ & $\begin{array}{l}\text { Amplicon } \\
\text { size (bp) }\end{array}$ \\
\hline GAPDH & NM_002046 & Hs00266705_g1 & 74 \\
HPRT1 & NM_000194 & Hs01003267_m1 & 72 \\
ACTB & NM_001101 & Hs01060665_g1 & 63 \\
PGP9.5 & NM_004181.3 & Hs00985157_m1 & 80 \\
\hline
\end{tabular}
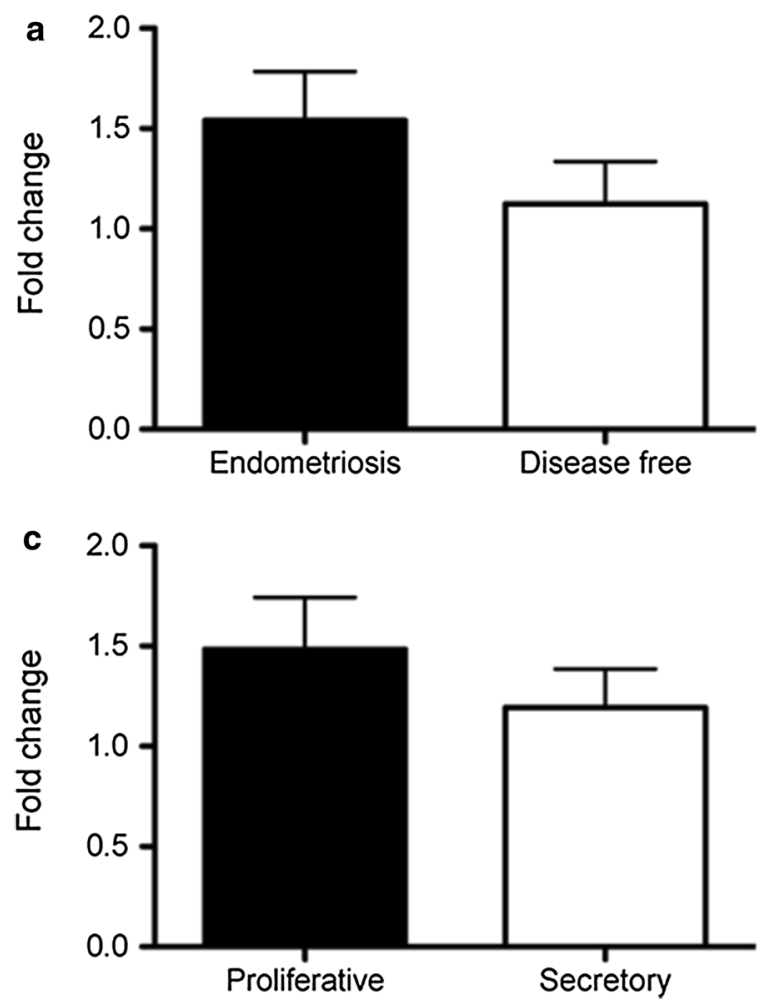

10 min followed by the addition of polyclonal rabbit antiPGP9.5 (dilution 1:1,400, incubated $30 \mathrm{~min}$, Dako A/S, Denmark). Following primary antibody incubation, the sections were further incubated with alkaline phosphatase/ RED, and streptavidin alkaline phosphatase (Dako, Denmark) for 15 min each. Staining was visualised with Fast Red chromogen (Dako) for $10 \mathrm{~min}$. Slides were counterstained with Mayer's haematoxylin (Fronine Pty, Australia) and blued in 2 dips of Scott's bluing solution, and then dried and mounted using Ultramount No 4 (Fronine Pty). Statistical analyses were conducted using independent samples Student's $t$ tests with an alpha value set at $P<0.05$ to explore differences between groups.

\section{Results}

Expression of PGP9.5 RNA in women with and without endometriosis

A comparison of PGP9.5 mRNA expression between women with endometriosis (1.541 \pm 0.243 , mean \pm SEM, $N=16)$ and without $(1.123 \pm 0.211, N=13)$ endometriosis found no significant difference $(P=0.220$, Fig. 1a). A similar analysis on PGP9.5 mRNA expression based on the pain reported and irrespective of the presence of
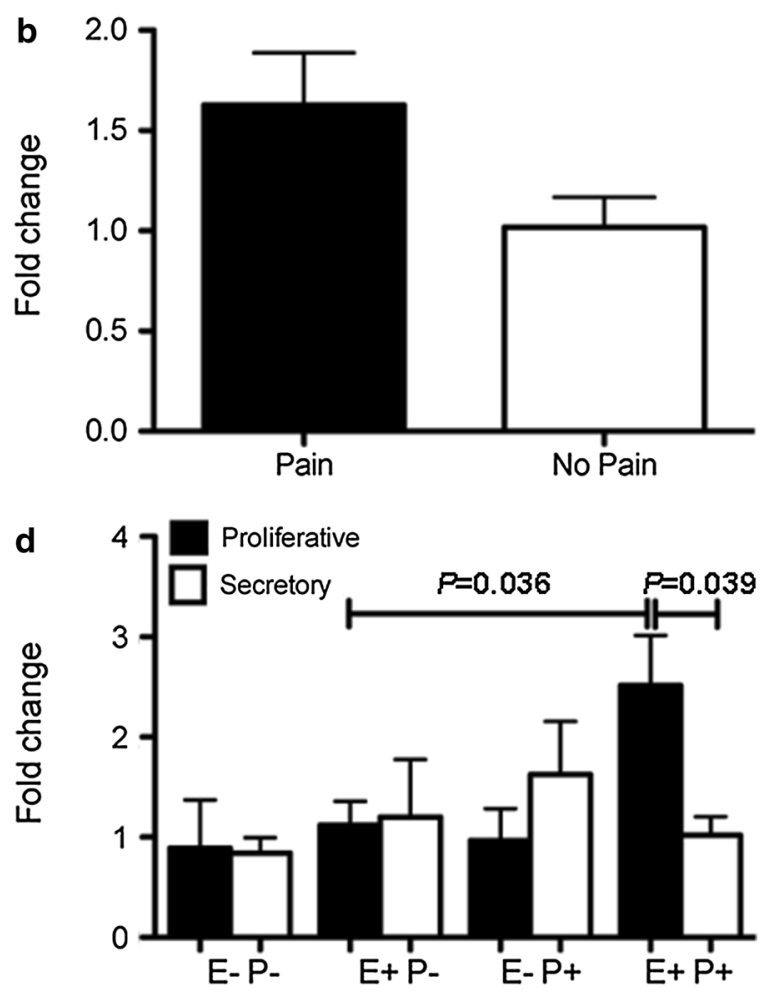

Fig. 1 Comparison of PGP9.5 mRNA expression based on the presence of endometriosis (panel a), pain (panel b), menstrual cycle phase (panel c) and in comparison with each other (panel d). Values are mean $\pm \mathrm{SEM}$ 
endometriosis or the menstrual cycle phase found that PGP9.5 gene expression was higher, although not quite at a level to reach statistical significance $(P=0.066)$ in women who reported pain $(1.627 \pm 0.259$ fold change compared to the calibration sample, $N=16$ ) compared to those who did not (1.017 \pm 0.151 fold change, $N=13)$ (Fig. 1b). A comparison between the menstrual cycle phases of all women also found no significant difference $(P=0.391)$ between proliferative $(1.485 \pm 0.257, N=16)$ and secretory phases $(1.193 \pm 0.193$ fold change, $N=13$ Fig. 1 c).

When all groups were stratified to include the three variables (endometriosis, pain and menstrual cycle phase) the largest expression of PGP9.5 mRNA occurred in women with endometriosis that reported painful symptoms and had samples taken during the proliferative phase $(2.514 \pm 0.499, N=5)$. The expression in this group was significantly higher than that observed in the similar group of women (with endometriosis and pain) but in the secretory phase $(1.018 \pm 0.187, N=4, \quad P=0.039)$. The expression in this group was also significantly higher than that observed when samples were taken in the proliferative phase of women with endometriosis, but without reported pain $(1.123 \pm 0.235, N=5, P=0.036$, Fig. 1 d).

Abundance of PGP9.5 protein in women with and without endometriosis

From the patients with endometriosis $(N=16)$ thirteen were positive (Fig. 2) for PGP9.5-immuno-reactivity in the endometrium ( $81 \%$ ); two with pain and one without. Conversely, none of the subjects without endometriosis showed immuno-reactivity against PGP9.5. A Student's $t$ test found no significant difference $(P>0.05)$ between

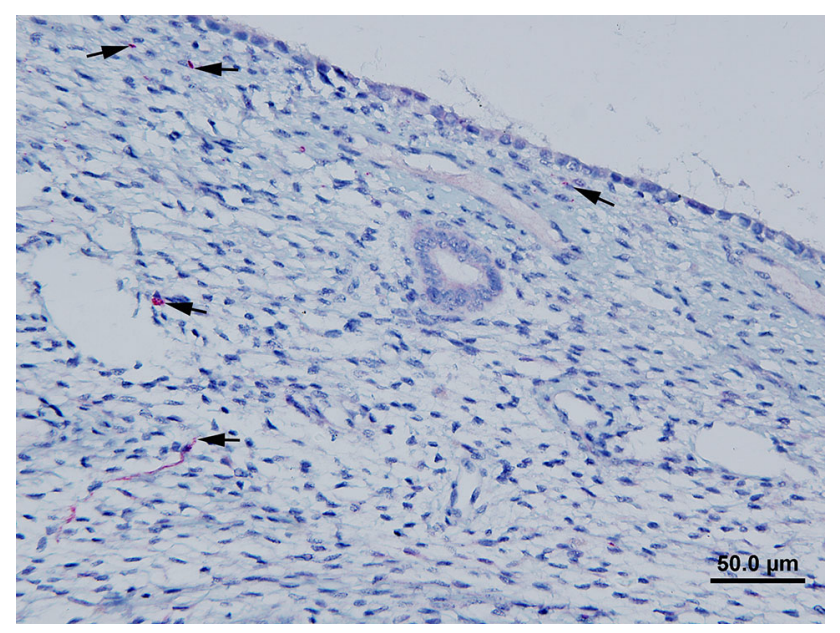

Fig. 2 Demonstration of PGP9.5 positive nerve fibres in the eutopic endometrium of a woman with endometriosis. Technical details are given in the "Materials and methods" section. The arrows indicate the signal for PGP9.5 the nerve fibre density in endometriotic women with pain (Mean $\pm \mathrm{SD} ; 1.8 \pm 0.6 \mathrm{~mm}^{2}$ ), compared to endometriotic women without pain $\left(1.6 \pm 1.8 \mathrm{~mm}^{2}\right)$.

When the patients in each group were divided between menstrual phase (secretory and proliferative) nerve fibre density was also similar. In the secretory phase, the patients with endometriosis and pain showed a mean of 1.8 nerve fibres per $\mathrm{mm}^{2}$, compared to 0.8 observed in the group of women with endometriosis and no pain. Correspondingly, the densities of nerve fibres in women with endometriosis in proliferative phase were 1.8 in the subgroup with pain vs. 2.4 in the subgroup without pain (Fig. 3). According to Student's $t$ tests, these differences between menstrual cycle phases were not statistically significant. Given that the large majority of the subjects had peritoneal endometriosis, it was not possible to assess a correlation between gene expression, protein abundance and the stage of the disease.

\section{Discussion}

This study includes only small sample numbers per group, but to our knowledge it is the first to investigate gene transcription as well as protein expression of the same marker and in the same patients, and also taking the menstrual cycle phase and pain levels into account. Our results indicate that the mRNA expression of PGP9.5 is significantly upregulated in the endometrium of women with painful endometriosis during the proliferative phase. IHC confirmed the presence of PGP9.5 protein in the endometrium of endometriosis patients, although a similar increase in PGP9.5 positive nerve fibre density was not observed in these women. Nerve fibres were absent in all women without endometriosis, which as such is in agreement with previous reports $[6,16,17]$. In another immunohistochemical study, PGP9.5 positive fibres were detected in the functional layer of the endometrium in women with pain symptoms, but not in those without-irrespective of the presence of endometriosis or adenomyosis [16, 18]. On the other hand, absence, partial or total, of positive fibres has also been reported for the eutopic endometrium of endometriosis patients (but was present in lesions [17]). It has to be taken into account that these variations could be due to varying IHC protocols; discordant results are now thought to be inherent to the technical difficulties of the IHC PGP9.5 assay which will need further optimisation in the future. Similarly, in a study using Western Blots, no differences between cases and controls could be observed for PGP9.5 and other neurogenic markers in eutopic endometrium [17, 19].

A difference seems to exist between the gene and the protein expression for PGP9.5; the former was upregulated during the proliferative phase in women who reported pain, 
Fig. 3 Mean density of nerve fibres per $\mathrm{mm}^{2}$ in patients with endometriosis with pain vs. without pain in a proliferative and $\mathbf{b}$ secretory phases
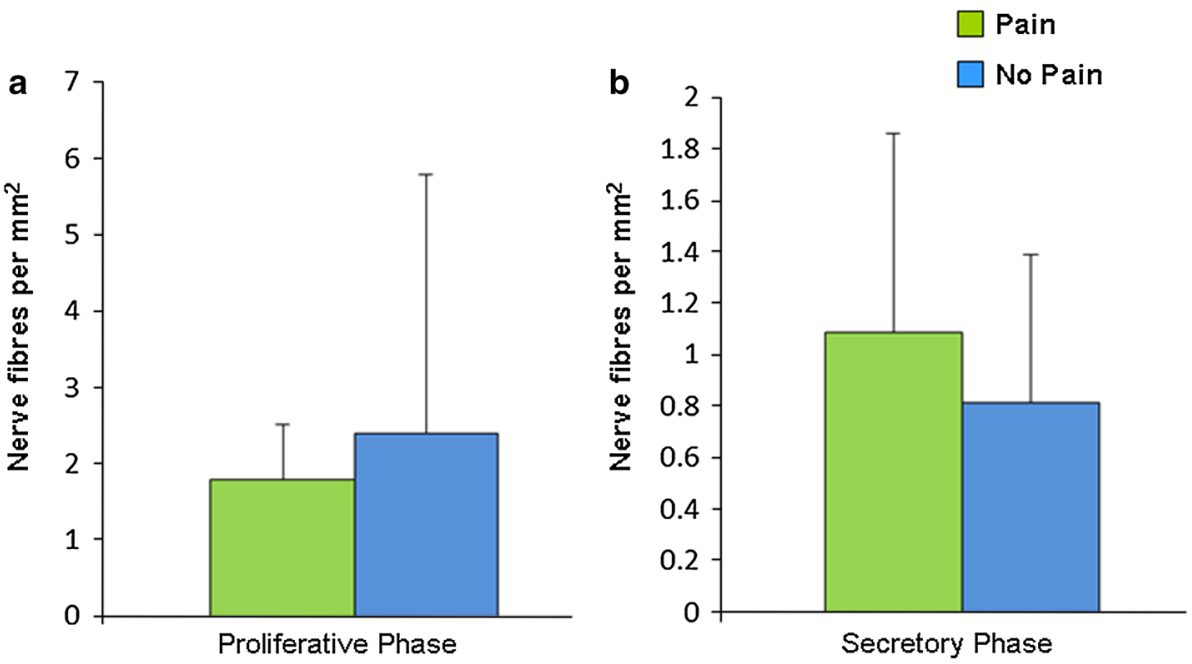

whereas the latter was correlated with the presence of endometriosis. These findings suggest that mechanisms regulating the translation of messenger RNAs and modification of assembled proteins could be differentially influencing the pathogenesis of endometriosis. Since the production of functional peptides and proteins is driven by gene expression it could be considered that gene regulation and differences in protein abundance correspond to each other in some ways within a specific tissue. Nevertheless, several other important regulatory events are involved in cellular processes following gene transcription and must be considered as possible further mechanisms for regulating the ultimate expression of individual proteins.

Recently, simultaneous microarray and proteome analyses in the same endometrial samples demonstrated overexpression of several genes [20]; however, the proteomic analysis showed no correspondence between these genes and the peptides reported to be dysregulated [21]. As in the previous study, this analysis did not find concordant overexpression of PGP9.5 at genetic and proteomic levels. Discordance between genomic and proteomic profiling is difficult to explain but a frequent phenomenon. Here, these discordances suggest that the expression of this protein is not only ruled by gene transcription, but additionally by regulatory mechanisms at the post-transcriptional level. A large number of mechanisms regulate gene expression, including mRNA transportation to the cytoplasm, codon phosphorylation and mRNA stability modifications, amongst others. mRNA stability is mediated to a large extent by endo- and exonucleases which cause polyA tail degradation, 5'-cap destruction and mRNA decay. The rates at which these processes occur are mediated by specialised proteins that attach to specific sequences in the $3^{\prime}$-untranslated region (3'-UTR) [22].

One important way cells regulate the abundance of certain proteins is through microRNAs (miRNAs). These molecules are short non-coding RNA sequences which are complementary to sequences located on the $3^{\prime}$-UTR of specific mRNA molecules [23]. When miRNAs are active, they associate with specific proteins to build RNA-induced silencing complexes, which include argonaute proteins, responsible to a great extent for the detection of mRNAs targeted for destruction [24].

Several microRNAs (miRNAs) have been identified in mammalian cells and some have been studied in the field of endometriosis (such as miRNA-135-a and -b, miRNA-202, miRNA-193a3p, miRNA-708 and mi-RNA-34c5p amongst others), with possible roles in post-transcriptional regulation of specific proteins presumably involved in processes of cell proliferation, apoptosis, tissue regeneration, inflammation and angiogenesis [25-32]. Even though a number of miRNAs involved in the signalling pathways associated with nerve growth have been reported to be dysregulated in women with endometriosis (i.e., miRNA29c, miRNA-125b, miRNA-126, miRNA-143 and miRNA34b targeting the genes PIK3R1, TNF, PIK3R2, MAPK7 and MAPK4, respectively) [26, 28], no miRNAs acting directly over PGP9.5 transcripts in endometriosis have been identified as yet in the literature. Furthermore, mRNA translation is regulated to some extent by RNA interference; this process is mediated by short lengths of doublestranded RNA called small interfering RNAs. These molecules attach to their matching mRNAs post-transcriptionally to degrade them, presumably by providing a scaffold for nucleases to attach and digest mRNA.

Bearing in mind the post-transcriptional mechanisms that could be altered more specifically in endometriosis, it could be likely that the level of proteins present in the endometrium at a specific point in time is higher than the number of transcripts of the corresponding gene; mRNA molecules are very unstable and have half-lives that range from 2 min to more than $10 \mathrm{~h}$, and generally an average of 
around $30 \mathrm{~min}$ in eukaryotic cells [22]. Considering these facts, it is possible that when certain proteins in the endometrium are detectable, the corresponding genes are already turned off and the mRNA molecules have already been degraded by ribonucleases for they would be no longer necessary. If this is true, up- and down-regulation should be evident at different periods; gene expression differences would occur earlier than protein abundance, even in the same specimen.

It has been reported that ubiquitin is more abundant in endometrial cells in the proliferative than in the secretory phase [33]. This would be in agreement with our finding of the increased expression of PGP9.5 gene in patients with pain in the proliferative phase when compared with the secretory phase, given that it is involved in deubiquitination from proteins marked for degradation by proteasomes [14]. Research on ubiquitin and endometriosis is very scant and thus it is hard to propose further associations with the disease pathogenesis, though it could be of interest to correlate the gene expression of ubiquitin and PGP9.5 in a continuation of this study with larger patient numbers. This would, however, still not explain the discordance between PGP9.5 gene expression and protein abundance found in this research-but this problem has been addressed above.

Further research, with study designs including genomic, proteomic and post-transcriptional regulatory approaches are therefore still necessary to address the discrepancies between gene and protein expression as well as the apparently inconsistent results for PGP9.5 protein production as a function of endometriosis and/or pain in the literature, and to identify factors and pathways that rule phenotypic features in women with endometriosis and provide potential therapeutic options to control disease development and progression.

Acknowledgments The immuno-histochemistry picture was provided by the courtesy of Dr. Frank Manconi, University of Sydney.

Conflict of interest The authors declare that they have no conflict of interest.

\section{References}

1. Chung SY, Kim SJ, Kim TH, Ryu WG, Park SJ, Lee DY et al (2005) Computed tomography findings of pathologically confirmed pulmonary parenchymal endometriosis. J Comput Assist Tomogr 29:815-818

2. Fraser IS (2008) Recognising, understanding and managing endometriosis. J Reprod Sci 1:56-64

3. Fraser IS (2010) Mysteries of endometriosis pain: Chien-Tien Hsu Memorial Lecture 2009. J Obstet Gynaecol Res 36:1-10

4. Tariverdian N, Theoharides TC, Siedentopf F, Gutierrez G, Jeschke U, Rabinovich $\mathrm{G}$ et al (2007) Neuroendocrine-immune disequilibrium and endometriosis: an interdisciplinary approach. Semin Immunopathol 29:193-210
5. Wang G, Tokushige N, Russell P, Dubinovsky S, Markham R, Fraser IS (2009) Hyperinnervation in intestinal deep infiltrating endometriosis. J Minim Invasive Gynecol 16:713-719

6. Tokushige N, Markham R, Russell P, Fraser IS (2006) High density of small nerve fibres in the functional layer of the endometrium in women with endometriosis. Hum Reprod 21:782-787

7. Tokushige N, Markham R, Russell P, Fraser IS (2006) Nerve fibres in peritoneal endometriosis. Hum Reprod 21:3001-3007

8. Tokushige N, Markham R, Russell P, Fraser IS (2008) Effects of hormonal treatment on nerve fibers in endometrium and myometrium in women with endometriosis. Fertil Steril 90:1589-1598

9. Al-Jefout M, Andreadis N, Tokushige N, Markham R, Fraser IS (2007) A pilot study to evaluate the relative efficacy of endometrial biopsy and full curettage in making a diagnosis of endometriosis by the detection of endometrial nerve fibres. Am J Obstet Gynecol 197:578-580

10. Al-Jefout M, Dezarnaulds G, Cooper M, Tokushige N, Luscombe GM, Markham R et al (2009) Diagnosis of endometriosis by detection of nerve fibres in an endometrial biopsy: a double blind study. Hum Reprod 24:3019-3024

11. Otsuki T, Yata K, Takata A, Hyodoh F, Miura Y, Sakaguchi H et al (2004) Expression of protein gene product 9.5 (PGP9.5)/ ubiquitin-C-terminal hydrolase 1 (UCHL-1) in human myeloma cells. Br J Haematol 127:292-298

12. Dhillon AP, Rode J, Dhillon DP, Moss E, Thompson RJ, Spiro SG et al (1985) Neural markers in carcinoma of the lung. Br J Cancer 51:645-652

13. Boudreaux D, Chaney J, Maiti TK, Das C (2012) Contribution of active site glutamine to rate enhancement in ubiquitin C-terminal hydrolases. FEBS J 279:1106-1118

14. Komander D, Clague MJ, Urbé S (2009) Breaking the chains: structure and function of the deubiquitinases. Nature 10:550-563

15. Campbell LK, Thoma JR, Lamps LW, Smoller BR, Folpe AL (2003) Protein gene product 9.5 (PGP 9.5) is not a specific marker of neural and nerve sheath tumors: an immunohistochemical study of 95 mesenchymal neoplasms. Mod Pathol 16:963-969

16. Bokor A, Kyama CM, Vercruysse L, Fassbender A, Gevaert O, Vodolazkaia A et al (2009) Density of small diameter sensory nerve fibres in endometrium: a semi-invasive diagnostic test for minimal to mild endometriosis. Hum Reprod 24:3025-3032

17. Donnez O, Soares M, Defrère S, Van Kerk O, Van Langendonckt A, Donnez J et al (2013) Nerve fibers are absent in disease-free and eutopic endometrium, but present in endometriotic (especially deep) lesions. J Endometr Pelvic Pain Disord 5:68-76

18. Zhang X, Lu B, Huang X, Xu H, Zhou C, Lin J (2009) Endometrial nerve fibres in women with endometriosis, adenomyosis, and uterine fibroids. Fertil Steril 92:1799-1801

19. Newman TA, Bailey JL, Stocker LJ, Woo YL, Macklon NS, Cheong YC (2013) Expression of neuronal markers in the endometrium of women with and those without endometriosis. Hum Reprod 28:2502-2510

20. Sherwin JR, Sharkey AM, Mihalyi A, Simsa P, Catalano RD, D'Hooghe TM et al (2008) Global gene analysis of late secretory phase, eutopic endometrium does not provide the basis for a minimally invasive test of endometriosis. Hum Reprod 23:1063-1068

21. Fassbender A, Simsa P, Kyama CM, Waelkens E, Mihalyi A, Meuleman C et al (2010) Trizol treatment of secretory phase endometrium allows combined proteomic and mRNA microarray analysis of the same sample in women with and without endometriosis. Reprod Biol Endocrinol 8:123. doi:10.1186/14777827-8-123

22. Alberts B, Johnson A, Lewis J, Raff M, Roberts K, Walter P (2008) Molecular biology of the cell. Garland Science, New York 
23. Lewin B (2008) Genes IX. Jones and Bartlett, Massachusetts

24. Pare JM, Lopez-Orozco J, Hobman TC (2011) MicroRNA-binding is required for recruitment of human argonaute 2 to stress granules and P-bodies. Biochem Biophys Res Comm 414:259-264

25. Pan Q, Luo X, Toloubeydokhti T, Chegini N, Pan Q, Luo X et al (2007) The expression profile of micro-RNA in endometrium and endometriosis and the influence of ovarian steroids on their expression. Mol Hum Reprod 13:797-806

26. Burney RO, Hamilton AE, Aghajanova L, Vo KC, Nezhat CN, Lessey BA (2009) MicroRNA expression profiling of eutopic secretory endometrium in women with versus without endometriosis. Mol Hum Reprod 15:625-631

27. Filigheddu N, Gregnanin I, Porporato PE, Surico D, Perego B, Galli L et al (2010) Differential expression of microRNAs between eutopic and ectopic endometrium in ovarian endometriosis. J Biomed Biotech 2010:369549. doi:10.1155/2010/ 369549

28. Teague EM, Print CG, Hull ML (2010) The role of microRNAs in endometriosis and associated reproductive conditions. Hum Reprod Update 16:142-165
29. Hawkins SM, Creighton CJ, Han DY, Zariff A, Anderson ML, Gunaratne PH et al (2011) Functional microRNA involved in endometriosis. Mol Endocrinol 25:821-832

30. Petracco R, Grechukhina O, Popkhadze S, Massasa E, Zhou Y, Taylor HS (2011) MicroRNA 135 regulates HOXA10 expression in endometriosis. J Clin Endocrinol Metab 96:E1925-E1933

31. Ramon LA, Braza A, Gilabert J, Gilabert J, Espana F, Chirivella $M$ et al (2011) MicroRNAs expression in endometriosis and their relation to angiogenic factors. Hum Reprod 26:1082-1090

32. Zhao ZZ, Croft L, Nyholt DR, Chapman B, Treloar SA, Hull ML et al (2011) Evaluation of polymorphisms in predicted target sites for micro RNAs differentially expressed in endometriosis. Mol Hum Reprod 17:92-103

33. Ilad RS, Fleming SD, Bebington CR, Murphy CR (2004) Ubiquitin is associated with the survival of ectopic stromal cells in endometriosis. Reprod Biol Endocrinol 2:69. doi:10.1186/14777827-2-69 